\title{
Characterizing Block Graphs in Terms of One-vertex Extensions
}

\author{
L. MARKENZON ${ }^{1 *}$ and C.F.E.M. WAGA ${ }^{2}$ \\ Received on February 19, 2018 / Accepted on February 22, 2019
}

\begin{abstract}
Block graphs has been extensively studied for many decades. In this paper we present a new characterization of the class in terms of one-vertex extensions. To this purpose, a specific representation based on the concept of boundary cliques is presented, bringing about some properties of the graph.
\end{abstract}

Keywords: block graph, one-vertex-extension.

\section{INTRODUCTION}

Block graphs have been extensively studied for many decades with characterizations based on different approaches since the first one in 1963 until today. In this paper we present a new characterization of the class in terms of one-vertex extensions. As block graphs are a subclass of chordal graphs, properties of this class can be successfully particularized: a specific representation of block graphs based on the concept of boundary cliques is presented, bringing about some properties of the graph.

Harary [7] introduced the definition of a block graph based on structural properties and presented a classical characterization: the block graph $B(G)$ of a given graph $G$ is that graph whose vertices are the blocks (maximal 2-connected components) $B_{1}, \ldots, B_{k}$ of $G$ and whose edges are determined by taking two vertices $B_{i}$ and $B_{j}$ as adjacent if and only if they contain a cut-vertex (its removal disconnects the graph) of $G$ in common. A graph is called a block graph if it is the block graph of some graph.

Characterization 1. [7] A graph is a block graph if and only if all its blocks are complete.

*Corresponding author: C. F. E. M. Waga - E-mail: waga@ime.uerj.br-https ://orcid.org/0000000234317730

${ }^{1}$ Programa de Pós-Graduação em Informática, Universidade Federal do Rio de Janeiro, Av. Athos da Silveira Ramos, 274, 21941-916, Rio de Janeiro, RJ, Brasil - E-mail: markenzon@nce.ufrj.br

${ }^{2}$ Departamento de Estruturas Matemáticas, IME, Universidade do Estado do Rio de Janeiro, R. São Francisco Xavier, 524, 20550-900, Rio de Janeiro, RJ, Brasil - E-mail: waga@ime.uerj.br 
Howorka [8], Bandelt and Mulder [2] and Behtoei et al. [3] presented characterizations based on metric conditions.

Characterization 2. [8] A graph is a block graph if and only if for every four vertices $u, v, w, x$, the larger two of the distance sums $d(u, v)+d(w, x), d(u, w)+d(v, x), d(u, x)+d(v, w)$ are equal.

Characterization 3. [2] A graph $G=(V, E)$ is a block graph if and only if for every vertices $u, v, w \in V, G$ satisfies

1. $I(u, v) \subseteq I(u, w) \cup I(v, w)$

2. $I(u, w) \subseteq I(u, v) \cup I(v, w)$

3. $I(v, w) \subseteq I(u, v) \cup I(u, w)$

where $I(x, y)=\{z \in V ; d(x, y)=d(x, z)+d(z, y)\}, x, y \in V$.

Characterization 4. [3] A graph $G$ is a block graph if and only if it satisfies:

1. the shortest path between any two vertices of $G$ is unique and

2. for each edge $e=u v \in E$, if $x \in N_{e}(u)$ and $y \in N_{e}(v)$, then, and only then, the shortest path between $x$ and $y$ contains the edge e, where $N_{e=u v}(v)=\{w \in V ; d(v, w)<d(u, w)\}$.

Bandelt and Mulder [1] presented a characterization based on forbidden subgraphs.

Characterization 5. [1] A graph is a block graph if and only if it is $C_{n \geq 4}$-free and diamond-free.

Mulder and Nebeský [11] characterized block graphs using an algebraic approach, a binary operation + (leap operation) on a finite nonempty set $V$ such that for $u, v, w \in V$,

1. $(u+v)+u=u$.

2. if $u \neq v$ then $(u+v)+v \neq u$.

3. if $u+v \neq v$ then $((u+v)+v)+u \neq u$.

4. if $u \neq v=u+v, u+w \neq v$ and $v+w \neq u$ then $u+w=v+w$.

The underlying graph $G_{+}=\left(V, E_{+}\right)$of + is such that $u v \in E_{+}$if and only if $u \neq v, u+v=v$ and $v+u=u$.

Intuitively, for any two vertices $u$ and $w$ in different blocks, the leap operation produces the cutvertex $z$ in the block of $u$ on the way to $w$, i.e., $u+w=z$. If $u$ and $w$ are in the same block, then $u+w=w$.

Characterization 6. [11] $G$ is a block graph if and only if it is the underlying graph of a leap operation on $V$. 
Recently, the subject was resumed. Dress et al. [5] characterized block graphs in terms of their vertex-induced partitions: any partition of a given finite set $V$ is a $V$-partition, and $P_{V}=\left(p_{v}\right)_{v \in V}$ is a $V$-indexed family of $V$-partitions. A family $P_{V}$ is a compatible family of $V$-partitions if, for any two distinct elements $u, v \in V$, the union of the set in $p_{v}$ that contains $u$ and the set in $p_{u}$ that contains $v$ coincides with the set $V$, and $\{v\} \in p_{v}$ holds for all $v \in V$. Let $P(V)$ denote the set of all compatible families of $V$-partitions.

Characterization 7. [5] There is a bijective function between the block graphs with vertex set $V$ and $P(V)$.

Mulder [10] presented a surprisingly simple characterization.

Characterization 8. [10] The graph $G$ is a block graph if and only if there exists a unique induced path between any two vertices in $G$.

\section{BACKGROUND}

Basic concepts about chordal graphs are assumed to be known and can be found in Blair and Peyton [4] and Golumbic [6]. In this section, the most pertinent concepts are reviewed.

Let $G=(V(G), E(G))$, or simply $G=(V, E)$, be a connected graph, where $|E|=m$ and $|V|=n$. The set of neighbors of a vertex $v \in V$ is denoted by $N_{G}(v)=\{w \in V ; v w \in E\}$ and its closed neighborhood by $N_{G}[v]=N_{G}(v) \cup\{v\}$. Two vertices $u$ and $v$ are true twins in $G$ if $N_{G}[u]=N_{G}[v]$ and false twins in $G$ if $N_{G}(u)=N_{G}(v)$. For any $S \subseteq V$, the subgraph of $G$ induced by $S$ is denoted $G[S]$. The set $S$ is a clique if $G[S]$ is complete. A vertex $v \in V$ is said to be simplicial in $G$ when $N_{G}(v)$ is a clique in $G$.

It is worth mentioning two kinds of cliques in a chordal graph $G$. A simplicial clique is a maximal clique containing at least one simplicial vertex. A simplicial clique $Q$ is called a boundary clique if there exists a maximal clique $Q^{\prime}, Q \neq Q^{\prime}$, such that $Q \backslash Q^{\prime}$ is a set of simplicial vertices of $G$.

A perfect elimination ordering (peo) of a graph $G=(V, E)$ is a bijective function $\sigma:\{1, \ldots n\} \rightarrow$ $V$ such that $\sigma(i)$ is a simplicial vertex in the induced subgraph $G_{i}=G[\{\sigma(i), \ldots, \sigma(n)\}]$, for $1 \leq i<n$. A peo is ultimately an arrangement of $V$ in a sequence $\sigma(V)=[\sigma(1), \ldots, \sigma(n)]$. It is well known that a graph $G$ is chordal if and only if $G$ admits a perfect elimination ordering.

\section{BOUNDARY REPRESENTATION}

In this section we present a representation of block graphs based on the concept of a perfect elimination ordering of the graph. As in a peo, where a vertex is eliminated when it is simplicial in the remaining graph, in this proposed representation, a maximal clique is eliminated when it is a boundary clique in the remaining graph. As all elements of the maximal clique are stored, the graph can be easily recovered. The representation is defined as follows; its structure is similar to the one presented in [9]. 
Let $G=(V, E)$ be a block graph with $\ell$ maximal cliques. A boundary representation of $G$ is the sequence of pairs

$$
B R(G)=\left[\left(P_{1}, s_{1}\right), \ldots,\left(P_{\ell}, s_{\ell}\right)\right]
$$

such that

- $P_{i} \cup\left\{s_{i}\right\}=Q_{i}, i=1, \ldots, \ell-1$, is a boundary clique of graph $G\left[V \backslash\left(Q_{1} \cup \ldots \cup Q_{i-1}\right)\right]$ where

- $P_{i} \subset V$ is the set of simplicial vertices of $Q_{i}$ and

- $s_{i} \in V$ is the cut-vertex of the clique $Q_{i}$;

- $P_{\ell}=Q_{\ell}$ is a maximal clique of $G$ and $s_{\ell}=\oslash$ (the symbol $\oslash$ denotes the absence of the parameter).

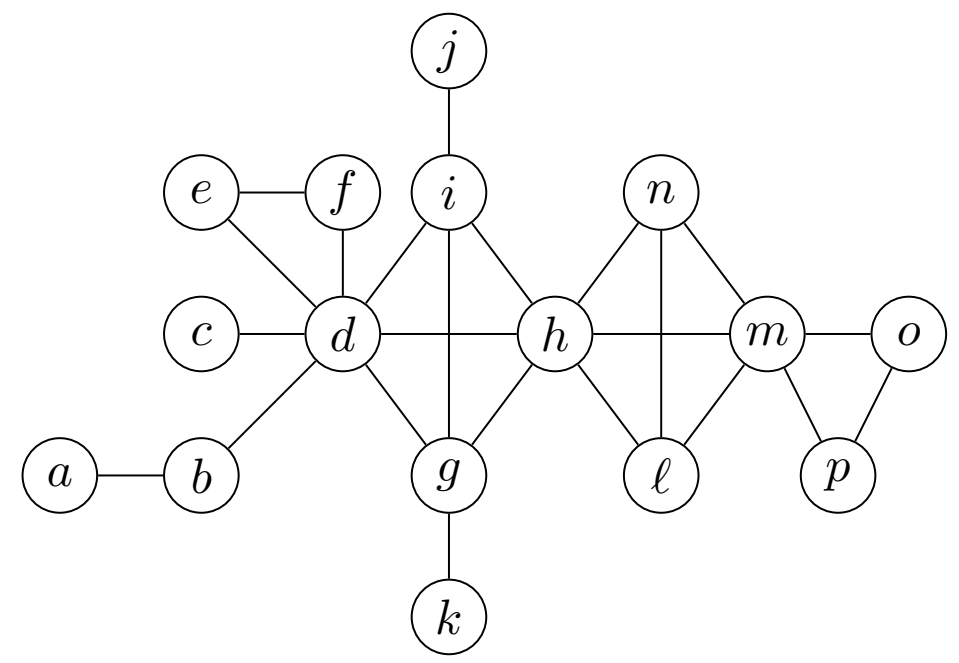

Figure 1: Block graph.

The algorithm to build the representation proceeds in stages. In each stage the boundary cliques of the current graph are determined. For each boundary clique, the simplicial vertices and its corresponding cut-vertex are recorded and all the simplicial vertices are removed from the graph. The process is repeated until only one clique remains. Note that it is possible to obtain a perfect elimination ordering of $G$ in direct sense, unlike other well known algorithms (lexicographic breadth-first search [12], for instance).

The boundary representation makes possible to deduce some structural properties of the graph.

Property 1. $P_{1} \cup\left\{s_{1}\right\}, P_{2} \cup\left\{s_{2}\right\}, \ldots, P_{\ell}$ are the maximal cliques of $G$.

Property 2. The set $\left\{P_{1}, \ldots, P_{\ell}\right\}$ is a partition of $V$. 
Property 3. The sequence provided by all vertices of $P_{1}$, followed by all vertices of $P_{2}$, and so on, up to $P_{\ell}$ is a perfect elimination ordering of $G$. Observe that, since there is no order in the set $P_{i}, i=1, \ldots, \ell$, several sequences can be built.

Employing the algorithm for the graph in Figure 1 we have:

$$
\begin{gathered}
B R(G)=[(\{a\}, b),(\{c\}, d),(\{e, f\}, d),(\{k\}, g),(\{j\}, i),(\{o, p\}, m),(\{b\}, d), \\
(\{m, n, \ell\}, h),(\{d, h, g, i\}, \oslash)]
\end{gathered}
$$

\section{ONE-VERTEX EXTENSIONS}

The concept of one-vertex extension was introduced by Bandelt and Mulder [1].

Let $G=(V, E)$ be a graph, $v \in V$ and $u \notin V$. An extension of $G$ to a graph $G^{\prime}=\left(V^{\prime}, E^{\prime}\right)$ is a one-vertex extension if it obeys one of the following three rules:

$(\alpha) V^{\prime}=V \cup\{u\}$ and $E^{\prime}=E \cup\{v u\}$, i.e., $N_{G^{\prime}}(u)=\{v\}$ (u is a pendant vertex).

$(\beta) V^{\prime}=V \cup\{u\}$ and $E^{\prime}=E \cup\left\{x u ; x \in N_{G}[v]\right\}$, i.e., $N_{G^{\prime}}[u]=N_{G^{\prime}}[v](u$ is a true twin of $v)$.

$(\gamma) V^{\prime}=V \cup\{u\}$ and $E^{\prime}=E \cup\left\{x u ; x \in N_{G}(v)\right\}$, i.e., $N_{G^{\prime}}(u)=N_{G^{\prime}}(v)(u$ is a false twin of $v)$.

The special cases of $(\alpha),(\beta)$ and $(\gamma)$ restricted to a simplicial vertex $v \in V$ are denoted by $\left(\alpha^{*}\right)$, $\left(\beta^{*}\right)$ and $\left(\gamma^{*}\right)$, respectively.

In order to generate a graph $G=(V, E)$, it is possible to establish a building sequence. A oneextension sequence (oes) of $G$ is a sequence of triples

$$
\Pi(G)=[\pi(1), \ldots, \pi(n)]
$$

being $\pi(i)=\left(e_{i}, v_{i}, u_{i}\right), i=2, \ldots, n$, such that

1. $e_{i} \in\left\{(\alpha),(\beta),(\gamma),\left(\alpha^{*}\right),\left(\beta^{*}\right),\left(\gamma^{*}\right)\right\}$;

2. $v_{i}=u_{j}$, for some $j<i$;

3. $u_{i} \neq u_{k}, 1 \leq k \leq i-1$;

and $\pi(1)$ is the special initial triple $\left(\oslash, \oslash, u_{1}\right)$.

Bandelt and Mulder [1] presented characterizations of distance hereditary graphs and ptolemaic graphs; the first one using the extensions $(\alpha),(\beta)$ and $(\gamma)$, and the second one using $(\alpha),(\beta)$ and $\left(\gamma^{*}\right)$. Theorem 4.1, presented below, shows a characterization of block graphs using one-vertex extensions.

Consider a graph $G, C V(G)$ the set of cut-vertices, $\operatorname{Simp}(G)$ the set of simplicial vertices and $\mathbb{Q}(G)$ the set of maximal cliques of the graph. 
Theorem 4.1. A graph $G=(V, E)$ is a block graph if and only if there is a sequence $\Pi(G)$ of $G$ composed by type $(\alpha)$ and type $\left(\beta^{*}\right)$ extensions.

Proof. Consider a block graph $G$ with $\ell$ maximal cliques and its boundary representation $B R(G)=\left[\left(P_{1}, s_{1}\right), \ldots,\left(P_{\ell}, \oslash\right)\right]$. It is possible to construct a sequence $\Pi(G)$ by transversing the boundary representation in reverse order.

Let $\left(P_{\ell}, \oslash\right), v \in P_{\ell}$ and $\pi(1)=(\oslash, \oslash, v)$. Consider $w \in P_{\ell} \backslash\{v\}$ and $\pi(2)=(\alpha, v, w)$. For $x \in$ $P_{\ell} \backslash\{v, w\}$, let be the triple $\left(\beta^{*}, w, x\right)$. So, there are the following elements of the sequence $\Pi(G)$ : $\pi(i)=\left(\beta^{*}, w, x\right), i=3, \ldots,\left|P_{\ell}\right|$. Thus, a first maximal clique of $G$ is obtained.

Let $\left(P_{\ell-j}, s_{\ell-j}\right), j=1, \ldots, \ell-1$. By the definition of boundary representation, $s_{\ell-j} \in Q_{k}, \ell-$ $j+1 \leq k \leq \ell$. Consider $v \in P_{\ell-j}$. The graph obtained by the extension $\pi\left(1+\sum_{k=\ell-j+1}^{\ell}\left|P_{k}\right|\right)=$ $\left(\alpha, s_{\ell-j}, v\right)$ has $v$ as a pendant vertex and $s_{\ell-j}$ as a cut-vertex. The vertices $v$ and $s_{\ell-j}$ belong to a new maximal clique $Q$. For $x \in P_{\ell-j} \backslash\{v\}$, let be the triple $\left(\beta^{*}, v, x\right)$. Thus, there are the following elements of the sequence $\Pi(G): \pi(i)=\left(\beta^{*}, v, x\right), i=2+\sum_{k=\ell-j+1}^{\ell}\left|P_{k}\right|, \ldots, \sum_{k=\ell-j}^{\ell}\left|P_{k}\right|$. These extensions increase the clique $Q$ to which vertex $v$ belongs in $G$. Then, we obtain the one-extension sequence of $G, \Pi(G)$, composed by type $(\alpha)$ and type $\left(\beta^{*}\right)$ extensions.

Conversely, consider $\Pi(G)=[(\oslash, \oslash, v)]$. The resulting graph $G$ is a trivial graph $(\{v\}, \emptyset)$ and it is a block graph.

Consider $H=(V(H), E(H))$ a block graph with $n-1$ vertices obtained by $\Pi(H)=$ $[\pi(1), \ldots, \pi(n-1)]$ a sequence of $(\alpha)$ and $\left(\beta^{*}\right)$ extensions. Let $v \in V(H), Q$ the maximal clique to which it belongs in $H$ and $u \notin V(H)$.

Let $\Pi(G)=\Pi(H) \| \pi(n)=[\pi(1), \ldots, \pi(n-1), \pi(n)]$.

If $\pi(n)=(\alpha, v, u)$, two cases must be analyzed.

1. $v$ is a simplicial vertex of $H$. Then, $C V(G)=C V(H) \cup\{v\}$ and $\operatorname{Simp}(G)=(\operatorname{Simp}(H) \backslash$ $\{v\}) \cup\{u\}$.

2. $v$ is a cut-vertex of $H$. Then, $C V(G)=C V(H)$ and $\operatorname{Simp}(G)=\operatorname{Simp}(H) \cup\{u\}$.

In both cases, the set of maximal cliques $\mathbb{Q}(G)=\mathbb{Q}(H) \cup\{v u\}$.

If $\pi(n)=\left(\beta^{*}, v, u\right), v$ must be a simplicial vertex in $H$. So, $C V(G)=C V(H), \operatorname{Simp}(G)=$ $\operatorname{Simp}(H) \cup\{u\}$ and $\mathbb{Q}(G)=(\mathbb{Q}(H) \backslash Q) \cup\left\{Q^{\prime}\right\}$ where $Q$ is a maximal clique such that $v \in Q$ and $Q^{\prime}=Q \cup\{u\}$.

In any case, $G$ is a block graph. 
The proof of Theorem 4.1 provides a possible one-extension sequence of a block graph. As an example, consider the block graph $G$ in Figure 1 and the boundary representation of the same graph presented in Section 3. The one-extension sequence obtained from $B R(G)$ is

$$
\begin{aligned}
\Pi(G)= & {\left[(\oslash, \oslash, d),(\alpha, d, h),\left(\beta^{*}, h, g\right),\left(\beta^{*}, h, i\right),(\alpha, h, m),\left(\beta^{*}, m, n\right),\right.} \\
& \left(\beta^{*}, m, \ell\right),(\alpha, d, b),(\alpha, m, o),\left(\beta^{*}, o, p\right),(\alpha, i, j),(\alpha, g, k), \\
& \left.(\alpha, d, e),\left(\beta^{*}, e, f\right),(\alpha, d, c),(\alpha, b, a)\right] .
\end{aligned}
$$

\section{ACKNOWLEDGEMENT}

This work was partially supported by CNPq grant 304706/2017-5.

RESUMO. Grafos bloco têm sido extensivamente estudados por muitas décadas. Neste artigo apresentamos uma nova caracterização da classe em termos de extensões em um vértice. Com esse objetivo, é definida uma representação especial, baseada no conceito de cliques limítrofes, ressaltando propriedades dos grafos bloco.

Palavras-chave: grafo bloco, extensão em um vértice.

\section{REFERENCES}

[1] H.J. Bandelt \& H.M. Mulder. Distance-hereditary graphs. Journal of Combinatorial Theory, Series B, 41 (1986), 182-208.

[2] H.J. Bandelt \& H.M. Mulder. Three interval conditions for graphs. Ars Combinatoria, 29B (1990), 213-223.

[3] A. Behtoei, M. Jannesari \& B. Taeri. A characterization of block graphs. Discrete Applied Mathematics, 158 (2010), 219-221.

[4] J.R.S. Blair \& B. Peyton. An introduction to chordal graphs and clique trees. In Graph Theory and Sparse Matrix Computation, IMA, 56 (1993), 1-29.

[5] A. Dress, K. Huber, J. Koolen, V. Moulton \& A. Spillner. Characterizing block graphs in terms of their vertex-induced partitions. Australasian Journal of Combinatorics, 66(1) (2016), 1-9.

[6] M.C. Golumbic. "Algorithmic Graph Theory and Perfect Graphs". Academic Press, New York, 2 ed. (2004).

[7] F. Harary. A characterization of block graphs. Canadian Mathematical Bulletin, 6(1) (1963), 1-6.

[8] E. Howorka. On metric properties of certain clique graphs. Journal of Combinatorial Theory, Series B, 27 (1979), 67-74.

[9] L. Markenzon, P.R.C. Pereira, C.F.E.M. Waga, C.V.P. Friedmann \& A. Lozano. An efficient representation of chordal graphs. Operations Research Letters, 41 (2013), 331-335. 
[10] H. Mulder. An observation on block graphs. Bulletin of the Institute of Combinatorics and its Applications, 77 (2016), 57-58.

[11] H. Mulder \& L. Nebeský. Leaps: an approach to the block structure of a graph. Discussiones Mathematicae Graph Theory, 26 (2006), 77-90.

[12] D.J. Rose, R.E. Tarjan \& G. Lueker. Algorithmic aspects of vertex elimination on graphs. SIAM Journal on Computing, 5 (1976), 266-283. 